Article

\title{
The Efficacy of Polymer Coatings for the Protection of Electroless Copper Plated Polyester Fabric
}

\author{
Alenka Ojstršek ${ }^{1, *}$, Natalija Virant ${ }^{1}$, Daryl Fox ${ }^{2}$ (), Latha Krishnan ${ }^{2}$ (i) and Andrew Cobley ${ }^{2}$ (i) \\ 1 Institute of Engineering Materials and Design, Faculty of Mechanical Engineering, University of Maribor, \\ Smetanova 17, 2000 Maribor, Slovenia; virant.natalija109@gmail.com \\ 2 The Functional Materials Research Group, Department of Engineering, Computing and the Environment, \\ Coventry University, Priory Street, Coventry CV1 5FB, UK; ac7922@coventry.ac.uk (D.F.); \\ ab9187@coventry.ac.uk (L.K.); aa2266@coventry.ac.uk (A.C.) \\ * Correspondence: alenka.ojstrsek@um.si; Tel.: +386-2-220-7935
}

Received: 6 May 2020; Accepted: 1 June 2020; Published: 3 June 2020

check for updates

\begin{abstract}
The selective metallisation of textiles is becoming a very important process in the development of electronic or e-textiles. This study investigated the efficacy of polymer coatings for the protection of copper $(\mathrm{Cu})$ conductive tracks electroless plated on polyester (PES) fabric against laundering and rubbing, without essentially affecting the physical-mechanical and optical properties of the base material. After the electroless deposition of a consistent layer of $\mathrm{Cu}$ onto PES, four polymers were applied individually by screen-printing or padding. The physical-mechanical characterisation of coated textiles revealed that polyurethane resin (PUR) and modified acrylate resin (AR) had little effect on the air permeability, tensile strength and breaking tenacity of the PES, as compared to silicone elastomer polydimethylsiloxane (PDMS) and epoxy resin (ER). On the other hand, PUR and PDMS had higher abrasion resistance and photo-stability under prolonged UV irradiation, as compared to $\mathrm{AR}$ and ER. In addition, freshly $\mathrm{Cu}$ plated samples were coated with polymers, washed up to 30 cycles and characterised by measuring their electrical resistivity, determination of colour changes and the examination of the surface morphology. Based on these results, PUR presented the most suitable protection of $\mathrm{Cu}$ tracks on PES, with the lowest impact on physical-mechanical properties. ER is not recommended to be used for protection of $\mathrm{Cu}$ tracks on fabrics, due to its rigidity, low photo-stability, washing and wear durability.
\end{abstract}

Keywords: PES fabric; electroless copper plating; conductive textile; polymers coatings; corrosion protection

\section{Introduction}

The rapid growth in wearable technology has seen an increased demand for improved integration of electronic circuitry into textiles. The introduction of electrical conductivity onto the surface of textiles leads to the production of high-added value textiles i.e., the dubbed electronic textiles (e-textiles), with applications in electronic sensors, data storage, optoelectronic and photonics. Such e-textiles have found use in diverse commercial sectors such as sport, protective clothing, healthcare and technical purposes [1]. This has often involved electronic components and circuit boards being attached to the textile, but this inevitably leads to a change in the feel and drape of a textile and increases its mass. With its high conductivity and ductility, copper $(\mathrm{Cu})$ is one of the most attractive options for e-textiles and application on the surface of flexible, substrates. Electroless $\mathrm{Cu}$ deposition is a well-known, additive approach to coat non-conductive (dielectric) substrates, such as printed circuit boards [2], textile [3], glass [4,5] and ceramics [6], with a conductive, metallic layer. Electroless plating is a controllable, stable process, which includes a source of metal ions, reducing agents, complexing agents, a stabilizer, 
a buffering agent, and a wetting agent; with temperature, concentration of the reducing agent and $\mathrm{pH}$ as the most influential factors [7]. A palladium catalysed, redox reaction takes place during electroless deposition, which reduces $\mathrm{Cu}$ ions at a regular rate to $\mathrm{Cu}$ metal, whilst formaldehyde is oxidised at high $\mathrm{pH}$ [2]. Once electroless copper deposition has occurred, it is essential to protect $\mathrm{Cu}$ tracks from oxidation that can lead to degradation of their electronic and mechanical properties in any aggressive environments they may encounter such as sweat or washing. In addition, the textiles themselves are subjected to processes and activities during their lifetime, such as washing, (tumble) drying, and flexing whilst also being exposed to a variety of conditions: humidity, extreme temperatures, irradiation by UV, rain, etc. Consequently, good adhesion between the metal layer and substrate, especially polyester, can be difficult to achieve $[8,9]$. The roughness of textiles can also pose a challenge to the uniform and continuousness coating, influencing the conductivity [10]. Thus, a stable protection of the coating is very important. Poor protection could cause the plated metal to peel off during wear and laundering, which limits the widespread application of wearable devices [11,12]. However, it is also important that whatever approach is used to protect the $\mathrm{Cu}$, it does not have a negative impact on the physical-mechanical and optical properties of the base textile, whilst, from an economic and ecologic perspectives, it must remain cost effective and nontoxic.

Considering different methods for the corrosion protection of metallic materials, the use of organic coatings is the most widely applied approach [13]. The mechanisms of corrosion protection by organic coatings can be described by distinguishing two types of coatings: (i) barrier coating and (ii) coatings containing substances, which are active chemically or electrochemically. A number of research papers report diverse encapsulants or casting compounds for the protection of conductive layers on flexible textile materials, ensuring their reliability and long-term performance (conductivity), depending on the type of textiles, the metal being protected and the application procedures. Kazani et al. improved the washing durability (after 20 washing cycles) of two silver inks on fourteen woven fabrics (cotton, viscose, polyester, polyamide), by laminating a thermoplastic polyurethane layer over the conductive silver [14]. Yang et al. investigated ultraviolet (UV)-curable polyurethane acrylate paste as the protective layers and Paul et al. applied two UV-curable polyurethane pastes as interface and encapsulation layers on polyester fabric (PES)/cotton fabrics, by means of the screen-printing process, resulting in waterproof and durable screen printed silver on textiles [15,16]. In another approach, Cho et al. used polyurethane-sealing onto $\mathrm{Cu} / \mathrm{Ni}$ electroless plated polyester fabric for e-textiles [7]. Govaert and Vanneste employed several techniques to protect a conductive layer consisting of carbon nanotubes i.e., direct coating, transfer coating and screen printing, for the application of water based polyacrylate binder [1]. To improve wear durability and adhesion at the interface between $\mathrm{Cu}$ film and PES fabric, Jiang et al. treated the $\mathrm{Cu}$-coated polyester fabric with two commercial solutions of polyester-polyurethane and aqueous acrylate in different concentrations, which also showed advantages in the resistance to aging [17]. However, there is very little reported work on the post treatment of $\mathrm{Cu}$-coated polyester fabric for the improvement of wear and washing durability.

This research is divided into two parts: (i) the application of four commonly used polymers (polyurethane, acrylate, epoxy and polydimethylsiloxane) on PES fabric using two application processes and the subsequent characterisation of the fabrics. The intention here was to evaluate the influence of individual coatings on the physical-mechanical properties of the fabric, as well as to assess the photo-stability of coatings against prolonged UV irradiation and its abrasion resistance against rubbing; (ii) the use of polymer coatings to protect electroless $\mathrm{Cu}$ plated PES fabric against oxidation (corrosion) after washing (up to 30 cycles) and any changes this may have on the electrical resistivity of the $\mathrm{Cu}$, its colour and the effect on the surface morphology of the $\mathrm{Cu}$ deposit by microscopic techniques. The polymers selected for this research meet specific requirements including reasonable price, low environmental impact, wide industrial applicability, good resistance against water, low temperature performance and good adhesion on diverse types of textile materials. In this way, the suitability of the employment of particular polymers for protection of subsequently $\mathrm{Cu}$ metallised textiles would be determined with potential applications in wearable electronics. 


\section{Experimental}

\subsection{Materials}

A series of trials were performed using an industrially bleached plain-weave $100 \%$ polyester fabric (PES) with a mass/unit area of $120.6 \pm 0.89 \mathrm{~g} / \mathrm{m}^{2}$ and a thickness of $0.29 \mathrm{~mm}$, warp density of 69 threads/cm and weft density of 31 threads/cm.

Paraffin wax (Merck KGaA, Darmstadt, Germany) was applied to the fabric, to ensure that copper was only plated onto the desired area of the fabric. A commercially available electroless copper process supplied by A-Gas Electronic Materials Ltd., Rugby, UK was utilised involving the following products. For the electroless copper pre-treatment: Conditioner 3320A, Circuposit Pre Dip 3340 and Circuposit Catalyst 3344. The electroless Cu plating solution was made using Circuposit $3350 \mathrm{M}-1$ (M-1), Circuposit 3350 A-1 (A-1), Cuposit Z-1 (Z-1) and Cuposit Y (Y). The pre-treatment and electroless solution were prepared according to the supplier instructions.

Four commercially available polymers were selected for the protection of conductive tracks, namely, Sylgard 184 silicone elastomer kit (polydimethylsiloxane-PDMS) that contains two chemicals, base and curing agent (mixed in ratio 10:1) supplied by Dow Corning, Midland, Michigan, US: Elpeguard SL 1305 AQ-ECO (waterborne polyurethane resin-PUR): Elpeguard SL 1307 FLZ-S (modified acrylate resin -AR) and: Elpecast Wepox VT 3000 (epoxy resin-ER) solvent-free 2-pack casting resin (component A: component B, mixed in ratio 2:1), supplied by Lackwerke Peters GmbH\&Co., Kempen, Germany. The main properties of these polymers are shown in Table 1.

Table 1. Properties of applied polymers *.

\begin{tabular}{|c|c|c|c|c|c|c|c|}
\hline \multirow{2}{*}{ Polymer } & \multirow{2}{*}{ Colour } & \multirow{2}{*}{ Transparency } & \multirow{2}{*}{$\begin{array}{l}\text { Viscosity } \\
(\mathrm{m} \mathrm{Pa} / \mathrm{s})\end{array}$} & \multicolumn{2}{|c|}{ Cure Time } & \multirow{2}{*}{ Pot Life (h) } & \multirow{2}{*}{$\begin{array}{c}\text { Dielectric Strength } \\
(\mathrm{kV} / \mathrm{mm})\end{array}$} \\
\hline & & & & At $25^{\circ} \mathrm{C}(\mathrm{h})$ & At $100^{\circ} \mathrm{C}(\mathrm{min})$ & & \\
\hline Polydimethylsiloxane (PDMS) & clear & high & 3500 & 48 & 35 & up to 2 & 19 \\
\hline Polyurethane resin (PUR) & colourless & high & 30 & 4 & 20 & up to 2 & 75 \\
\hline Modified acrylate resin (AR) & fluorescent & high & low & - & 5 & 1 & 60 \\
\hline Epoxy resin $(\mathrm{ER})$ & yellowish & high & 1500 & 72 & 45 & 1.5 & 45 \\
\hline
\end{tabular}

* Data provided by the supplier (Technical reports).

\subsection{Selective Metallisation of Fabric by Electroless Copper Plating}

A PES fabric sheet was sectioned into samples of size $7 \times 4 \mathrm{~cm}^{2}$. The fabric sample was brushed with molten paraffin wax solution at a temperature of $65^{\circ} \mathrm{C}$, which left only the desired section of the textile, with an area of $4 \times 4 \mathrm{~cm}^{2}$, exposed for $\mathrm{Cu}$ plating. Electroless $\mathrm{Cu}$ plating was conducted as outlined in the steps of Figure 1. After drying the fabric sample at RT, it was dipped into the conditioner solution ( $450 \mathrm{~mL}$ deionised (D.I.) water with $50 \mathrm{~mL}$ conditioner) at $50{ }^{\circ} \mathrm{C}$ for $5 \mathrm{~min}$, which cleaned and functionalised the fabric' surface. Subsequently, the sample was rinsed thoroughly with the running tap water for $5 \mathrm{~min}$, and further dipped into the pre-dip solution (420 mL D.I. water with $135 \mathrm{~g}$ of pre-dip) for $1 \mathrm{~min}$ at RT. In addition, the fabric was immersed into the palladium (Pd) catalyst solution ( $420 \mathrm{~mL}$ of D.I. water with $135 \mathrm{~g}$ of pre-dip and $15 \mathrm{~mL}$ of Pd catalyst) for $5 \mathrm{~min}$ at $40^{\circ} \mathrm{C}$. The sample was then rinsed in water for $5 \mathrm{~min}$ and placed in to an electroless $\mathrm{Cu}$ bath, which was prepared as follows (for $500 \mathrm{~mL}$ ): $405 \mathrm{~mL}$ D.I. water, $75 \mathrm{~mL}$ of M-1, $5.0 \mathrm{~mL}$ of A-1, $5.25 \mathrm{~mL}$ of Z-1 and 7.5 mL of Y. The bath was heated to $46^{\circ} \mathrm{C}$ and the sample was immersed into the solution for $25 \mathrm{~min}$. The metallised fabric was finally rinsed in D.I. water for $5 \mathrm{~min}$, with the aim to remove excess plating solution, and dried in an oven $\left(40^{\circ} \mathrm{C}\right.$, typically overnight). The wax from the fabric was removed using ice water and then the fabric was washed in water at $60^{\circ} \mathrm{C}$, for the removal of the last traces of wax. The sample was wrapped in an aluminium foil and stored in a vacuum desiccator for characterisation. 


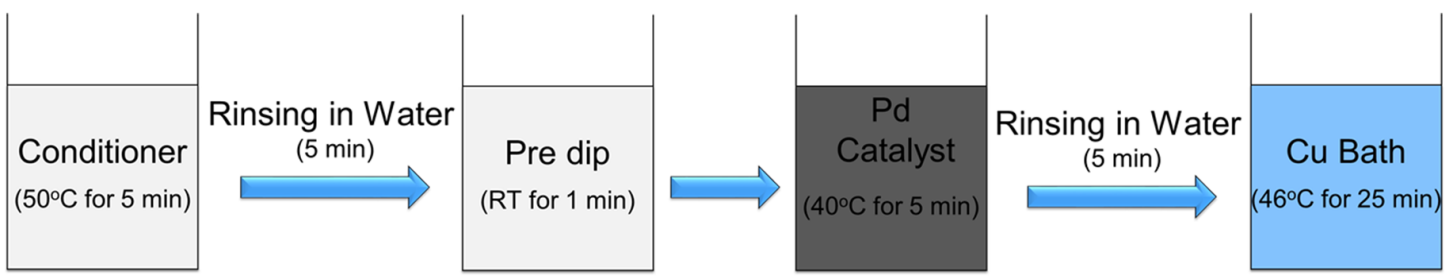

Figure 1. Scheme of the steps involved in an electroless copper $(\mathrm{Cu})$ plating of polyester $(\mathrm{PES})$ fabric.

\subsection{Application of Polymer Protective Coatings}

Two industrially applicable processes, i.e., screen-printing and padding, were used for the application of the protective coatings on textile material, due to significantly different viscosities of the four selected polymers.

PDMS and ER were applied onto fabrics using a screen-printing procedure, by means of a semiautomatic printing table Mini-MD (Johannes Zimmer, Klagenfurt, Austria). In order to achieve an even coverage of the polymer over the entire sample surface, the screen-printing frame with a PES 125 mesh stencil was placed on uncoated or metallised samples. The stainless-steel rod-roll squeegee was always rectangular on a warp direction of fabric. Pre-optimised parameters were used during printing, i.e., roll-rod diameter of $15 \mathrm{~mm}$, speed of $1 \mathrm{~m} / \mathrm{min}$, max magnetic pressure and two application layers.

PUR and AR were applied onto PES during the padding procedure, according to the previously optimized method. The sample (in a warp direction) was padded twice with PUR or AR dispersion, using a two-roll laboratory pad-mangle (Werner Mathis AG, Oberhasli, Switzerland) with a horizontal position of the squeezing rollers. The padding rate was $0.8 \mathrm{~m} / \mathrm{min}$, with the squeezing roller pressure of 2 bars along the roller contact line ( $80 \%$ wet pick-up). The padding was repeated twice, without intermediate drying.

After the application of the polymers, coated samples were dried in an oven at a temperature of $100^{\circ} \mathrm{C}$ for the time recommended by the polymer supplier (Table 1), in order to form a sufficient cross-linked network. The add-on percentage of the individual polymer on the surface of PES fabric was calculated after application according to Equation (1):

$$
\text { Add }- \text { on }=\frac{m_{\mathcal{C}}-m_{i}}{m_{i}} \times 100(\%)
$$

where $m_{c}$ is a mass of coated PES sample; and $m_{i}$ is a mass of PES before coating.

\subsection{Washing}

In order to evaluate the washing durability of the polymers applied onto PES, and consecutively, their protection ability against corrosion of $\mathrm{Cu}$ tracks, $\mathrm{Cu}$-metallised and protected samples were washed up to 30 times in a Labomat (Werner Mathis AG, Oberhasli, Switzerland), according to standard ISO $105-\mathrm{C} 06$, at a temperature of $40{ }^{\circ} \mathrm{C}$ for $30 \mathrm{~min}$, using a solution of $1 \mathrm{~g} / \mathrm{L}$ of standard reference detergent without optical brighteners, and a liquor-to-fabric weight ratio of 50:1. After each washing cycle, the samples were rinsed three times under tap water, where each rinsing cycle lasted for $1 \mathrm{~min}$, and then dried at room temperature. Electrical resistivity and CIE colour measurement of the samples were carried-out before and after a selected set of washing cycles; electrical resistivity was measured after 5, 10, 20 and 30 cycles, and colour after 5, 15 and 30 cycles. In addition, the changes in surface morphologies of samples before and after the washing tests were inspected by optical microscopy $(\mathrm{OM})$ and scanning electron microscopy (SEM). 


\subsection{Analytical Methods}

\subsubsection{Characterization of Polymers' Coated Textiles}

The physical-mechanical properties, air-permeability, abrasion resistance and photo-stability of polymer coated PES fabric were determined. This part of the study investigated the effect of the four polymers on the physical-mechanical and optical properties of a base textile material. All samples were conditioned before testing for $24 \mathrm{~h}$ in a standard atmosphere in climatic chamber, according to ISO/R 139 at temperature of $20 \pm 2{ }^{\circ} \mathrm{C}$ and relative humidity of $65 \pm 5^{\circ} \mathrm{C}$.

The thickness of un-coated and coated samples was determined according to standard ISO 5084, using universal thickness gauge meter (Luis Schoppen, Leipzig, Austria). Each sample was measured five times and the average value was calculated.

The air permeability of the fabric, before and after polymer coating, was carried out on five different places on each sample, according to standard EN ISO 9237, using a Karl Schröder KG device (Weinhem, Germany).

Selected mechanical properties, such as elongation at break, tensile strength and breaking tenacity of uncoated and polymers' coated samples were determined, according to Standard ISO 13934-1, using a Textechno statigraph M test machine (Textechno H. Stein GmbH \& Co. KG, Moenchengladbach, Germany). A total of five measurements were taken separately in both weft and warp directions for each sample $\left(25 \times 5 \mathrm{~cm}^{2}\right)$, in order to obtain statistically significant results.

The abrasion resistance of polymers' coatings on fabric was accomplished by the Martindale method, according to standard EN ISO 12947-3, using up to 20,000 rubbing cycles. The mass loss of the samples was determined after each set of rubbing cycles $(5,000,10,000$ and 20,000), according to ISO 3801 standard, using a Zweigle KG device (Zweigle Textilprüfmaschinen GmbH \& Co KG, Reutlingen, Germany).

For the evaluation of the photo-stability of the protective polymers, selected samples were irradiated with an artificial UV source for a prolonged time (up to 30 days). Sample sizes of $8 \times 5 \mathrm{~cm}$ were placed in a Luzchem photo-reactor (Luzchem Research Inc., Ottawa, Ontario, Canada), equipped with six overhead UV lamps, of which three lamps providing UV-A light within the range of 316-400 nm, with the main peak at $350 \mathrm{~nm}$, and three lamps emitting mainly UV-B light within the range of $281-315 \mathrm{~nm}$, with the main peak at $313 \mathrm{~nm}$. The UV luminance in the reactor was 18,600 Lux, measured by a Sperry Instruments Digital Light Meter, SLM-110 (ECM Industries, LLC, New Berlin, USA). The time-dependent stabilities of the applied polymers towards UV-provoked changes in fabrics' whiteness/yellowness were calculated after 30 days of irradiation (Equations (2) and (3)) from the reflectance measurements obtained by two-rays spectrophotometer Spectraflash SF 600 PLUS (Datacolor, Lawrenceville, New Jersey, USA), as described in Section 2.5.2.

$$
W=Y+800 \cdot\left(x_{n}-x\right)+1700 \cdot\left(y_{n}-y\right)
$$

where $W$ is the whiteness according to CIE; $Y$ is the tristimulus value; $x$ and $y$ are the chromaticity coordinates of the observed white sample; and $x_{n}$ and $y_{n}$ are chromaticity coordinates of the completely opaque standardized white object.

$$
Y I=\frac{100 \cdot\left(C_{X} \cdot X-C_{Z} \cdot Z\right)}{Y}
$$

where $Y I$ is the yellowness indices according to ASTM E313 method at D65/10 ; $C_{X}$ and $C_{Z}$ are coefficients, depend on illuminant and observer; $Y, X$ and $Z$ are tristimulus values in CIE colour space. 


\subsubsection{Analysis of Conductive Textiles}

Electrical resistivity, reflectance and colour of $\mathrm{Cu}$ metallised/polymer coated PES fabrics samples were determined after 30 washing cycles and the surface morphology was inspected, in order to study the suitability of selected polymers for the corrosion protection of $\mathrm{Cu}$ tracks on textiles.

The electrical resistance of Cu-metallised and Cu-metallised/protected samples was measured with the use of a set of standardized measuring electrodes at three measuring points/distances on each test sample (Figure 2), using a 34410A $6 \frac{1}{2}$ Digital Multimeter (Agilent Technologies, Santa Clara, USA).

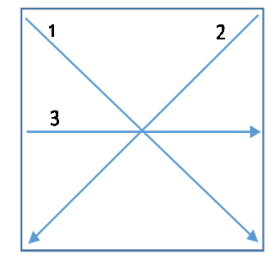

Figure 2. Electrical resistivity measuring lines/distances; $1(5.7 \mathrm{~cm}), 2(5.7 \mathrm{~cm})$ and $3(4 \mathrm{~cm})$.

Reflectance measurements of metallised and metallised/polymer coated samples were achieved within a spectral range of 400-700 nm wavelengths, by a two-rays spectrophotometer Spectraflash SF 600 PLUS (Datacolor, Lawrenceville, New Jersey, USA), equipped with an Ulbricht sphere and measuring geometry of $\mathrm{d} / 8^{\circ}$ under a standard illuminant D65 (LAV/Spec. Incl.), from which the CIE $\mathrm{L}^{*} \mathrm{a}^{*} \mathrm{~b}^{*}$ colour values were calculated, using Datacolor QC 600, V3.3 software. In addition, the CIE total colour differences $\left(d E^{*}\right)$ of $\mathrm{Cu}$ metallized/polymer coated samples before and after washing cycles were calculated from the coordinate differences in all three directions of the colour space by following Equation (4) [18]:

$$
d E *=\sqrt{(d L *)^{2}+(d a *)^{2}+(d b *)^{2}}
$$

where $d E^{*}$ is the total colour difference; $d L^{*}$ is the difference in brightness; $d a^{*}$ is the difference at the red/green axis; and $d b^{*}$ is the difference at the yellow/blue axis.

The surface appearance of $\mathrm{Cu}$-metallised and $\mathrm{Cu}$-metallised/coated fabrics before and after 30 washing cycles, as well as the cross-sectional appearance of $\mathrm{Cu}$-metallised/coated fabrics after 30 washings, were observed by Axiotech $25 \mathrm{HD}$ (+pol) Zeiss optical microscope (Carl Zeiss NTS GmbH, Oberkochen, Germany), equipped with an Axiocam MRc (D) high-resolution camera. All measurements were accomplished in light transmission mode, with a halogen light as the light source, using $10 \times$ magnification.

In addition, surface morphologies of $\mathrm{Cu}$-metallised and $\mathrm{Cu}$-metallised/coated fabrics after 30 washing cycles were inspected by attaching approximately $1 \mathrm{~cm}^{2}$ of fabric sample onto an adhesive carbon tape fixed to a brass holder and observed using a Zeiss Gemini Supra 35 VP Scanning Electron Microscope (Carl Zeiss NTS GmbH, Oberkochen, Germany).

\section{Results and Discussion}

\subsection{Characterization of Polymers' Coatings}

The aim of the first part of the study was to determine if four polymers, selected for their ability to protect copper from corrosion, would influence the physical-mechanical properties of the base textiles. As stated in the methodology, the PES fabric was firstly coated with an individual polymer using pre-optimized application procedures, and characterised. The obtained results are shown in Tables 2 and 3. In addition, the durability of the polymer-coated PES against rubbing was studied by determination of the mass loss (Figure 3), and the photo-stability under prolonged UV-rays exposure by reflectance measurement, from which the yellowness and whiteness indices were calculated (Figure 4). 
Table 2. Add-on, thickness and air permeability of coated PES.

\begin{tabular}{|c|c|c|c|c|c|c|}
\hline \multirow{2}{*}{ Sample } & \multirow{2}{*}{ Process Employed } & \multicolumn{2}{|c|}{ Drying } & \multirow{2}{*}{ Add-on (\%) } & \multirow{2}{*}{ Thickness (mm) } & \multirow{2}{*}{ Air Permeability $\left(\mathrm{L} / \mathrm{m}^{2} \mathrm{~s}\right)$} \\
\hline & & $T\left({ }^{\circ} \mathrm{C}\right)$ & Time (min) & & & \\
\hline reference & - & - & - & - & 0.289 & $228.4 \pm 9.5$ \\
\hline PDMS & Screen-printing & 100 & 25 & 39.26 & 0.323 & $47.8 \pm 2.4$ \\
\hline PUR & Padding & 100 & 20 & 25.38 & 0.314 & $215.4 \pm 13.6$ \\
\hline $\mathrm{AR}$ & Padding & 100 & 5 & 28.23 & 0.318 & $194.5 \pm 12.2$ \\
\hline ER & Screen-printing & 100 & 45 & 42.18 & 0.346 & $<5$ \\
\hline
\end{tabular}

Table 3. Electrical resistivity before and after washings.

\begin{tabular}{|c|c|c|c|c|c|c|c|c|c|c|c|c|c|c|c|c|c|c|}
\hline \multicolumn{19}{|c|}{ Electrical Resistivity $[\Omega]$} \\
\hline \multirow{2}{*}{\multicolumn{3}{|c|}{ Cu Metallized }} & \multirow{2}{*}{\multicolumn{4}{|c|}{ Cu Metallized/Polymer Coated }} & \multicolumn{12}{|c|}{ Washing Cycles } \\
\hline & & & & & & & \multicolumn{3}{|c|}{5} & \multicolumn{3}{|c|}{10} & \multicolumn{3}{|c|}{20} & \multicolumn{3}{|c|}{30} \\
\hline 1 & 2 & 3 & Polymer & 1 & 2 & 3 & 1 & 2 & 3 & 1 & 2 & 3 & 1 & 2 & 3 & 1 & 2 & 3 \\
\hline 0.4 & 0.2 & 0.1 & - & & & & 20.5 & 37.4 & 35.5 & $\begin{array}{c}46.2 \\
\mathrm{k}\end{array}$ & $\begin{array}{c}1 \\
M\end{array}$ & $\begin{array}{c}68.7 \\
\mathrm{k}\end{array}$ & - & - & - & - & - & - \\
\hline 0.8 & 0.4 & 0.3 & PDMS & 1.2 & 0.5 & 1.8 & 1.5 & 0.6 & 2.0 & 1.8 & 0.7 & 2.5 & 1.8 & 1.3 & 3.4 & 5.8 & 1.5 & 55 \\
\hline 0.2 & 0.2 & 0.9 & PUR & 2.5 & 2.3 & 2.9 & 7.5 & 3.5 & 6.9 & 12.7 & 5.8 & 14 & 17.2 & 12.2 & 16 & 22 & 26 & 15.5 \\
\hline 0.2 & 0.2 & 1.2 & AR & 1.7 & 0.2 & 1.5 & 2.5 & 0.4 & 5.8 & 2.8 & 0.8 & 6.1 & 2.8 & 0.8 & 6.5 & 2.9 & 0.9 & 12.2 \\
\hline 0.3 & 0.6 & 1.5 & ER & 3.7 & 7.1 & 7.5 & 7.8 & 5.9 & 2.7 & $\begin{array}{c}11.8 \\
\mathrm{k}\end{array}$ & $\begin{array}{c}17.4 \\
\mathrm{k}\end{array}$ & $\begin{array}{c}65.3 \\
\mathrm{k}\end{array}$ & - & - & - & - & - & - \\
\hline
\end{tabular}
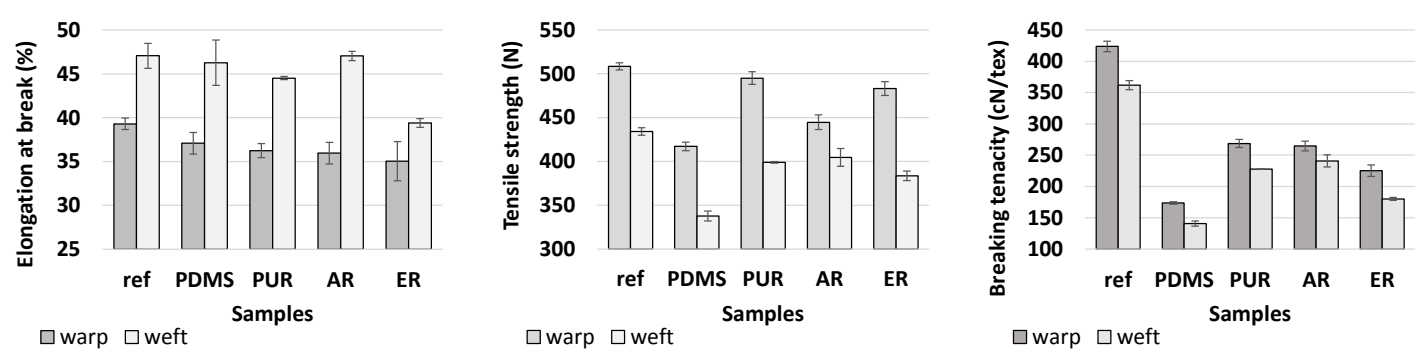

Figure 3. Mechanical features of uncoated and coated samples in warp and weft directions.

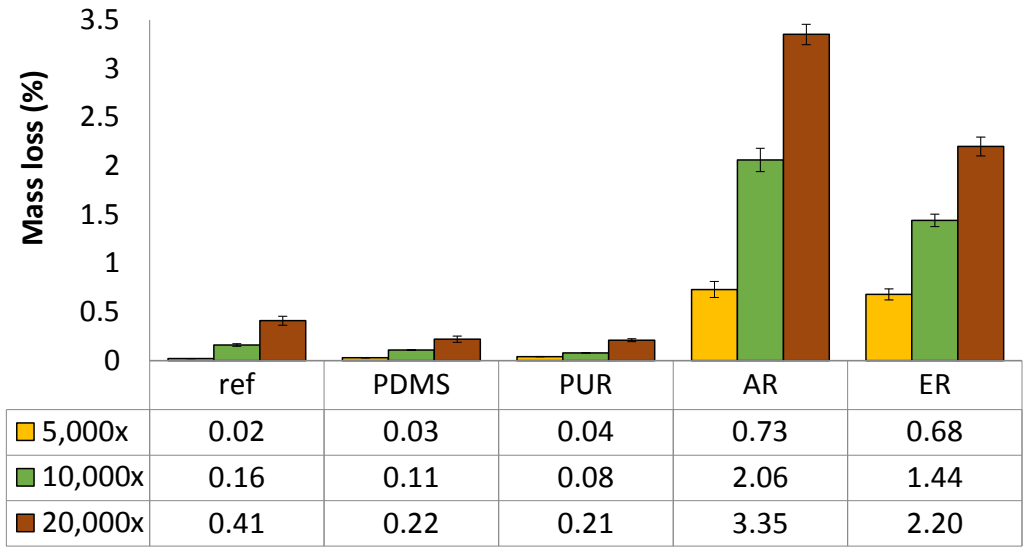

Figure 4. Mass loss of polymer coated PES samples, measured at 5,000, 10,000 and 20,000 rubbing cycles.

\subsubsection{Add-on, Thickness and Air Permeability}

Table 2 shows the percentage of applied coatings on the fabrics, and consecutively, thickness and air permeability, depending on the type of polymer and process employed.

From Table 2, it is evident that coated samples showed weight increments between $25.38 \%$ up to $42.18 \%$, depending on the applied polymer. AR and PUR polymers with the lower viscosities penetrate more easily into a textile structure than ER and PDMS with higher viscosities, which agrees with the results obtained by other researchers $[14,19]$. The air permeability of un-treated PES fabric was high, $228.4 \pm 9.5 \mathrm{~L} / \mathrm{m}^{2} \mathrm{~s}$, as airflow can simply penetrate through the pores of the fabrics. The application of polymers blocked the pores between the fibres and yarns, and thus, the fabrics' air permeabilities was reduced. This could also affect the thermal and moisture behaviour of the fabrics, 
if applied on larger surfaces, as suggested by Moiz et al. [20]. In our study, the highest add-on (42.18\%), and consecutively, the lowest air permeability (under the limit of detection) were determined for the ER screen-printed sample. This was due to the fact that ER did not penetrate to the inner parts of the fabric, but remained on the surface, forming a thick, rigid layer, as could also be observed from the OM and SEM images. PUR and AR reduced the air permeability to a negligible extent, and therefore, they are more appropriate for the application on textiles, from the comfort viewpoint, in comparison with PDMS and ER.

\subsubsection{Mechanical Features}

Mechanical properties are one of the important evaluations criteria of the performance of polymer coatings. It is expected that coatings tightly adhere to the fibre surface, ensuring their durability. At the same time, they should possess strength and flexibility for providing protection and extensibility. In this research, elongation at break, tensile strength and breaking tenacity of coated samples were compared with reference to uncoated PES, in both warp and weft directions. This enabled an evaluation of any possible negative effects of the employed polymers on the fabrics' mechanical properties. The obtained results were disclosed in Figure 3.

The consideration of Figure 3 indicates that, after application of polymers, the elongation at break and tensile strength were decreased moderately, compared to the reference sample, i.e., elongation at break by $3-11 \%$ (warp) and $0.04-16 \%$ (weft), and tensile strength by $3-18 \%$ (warp) and $7-22 \%$ (weft). This could be explained by the fact that the coating penetrates the fabric sample and inhibits the mobility of the yarns in the fabric structure, causing the fabric to become more rigid and inflexible [21,22]. The fabric rigidity depends also on the characteristics of the polymers used, the application conditions, and undesired linkages between the coated fibres, so-called bridges. In addition, the breaking tenacity drastically decreased, especially using PDMS as a coating polymer (for 59\%). Due to the restriction of warp and weft yarn movement in fabric structure, the fabric breaks as a whole (the yarns break at one time), as explained in detail by Bulut and Suülar [22].

\subsubsection{Abrasion Resistance}

The durability of textiles against rubbing is a significant parameter in everyday life (abrasion between textile and skin, textile and textile, textile and surrounding material), and it is therefore important that it should be determined. Moreover, the mechanical damage of polymer coatings could lower the protection of $\mathrm{Cu}$ on the surface of textile material, leading to oxidation of the $\mathrm{Cu}$ deposit. Thus, a standard abrasion resistance Martindale method was used, where PES samples coated with the selected four polymers were exposed to 5,000,10,000 and 20,000 rubbing cycles. The mass loss after each set of rubbing cycles was determined (Figure 4).

From Figure 4, it is observed that uncoated PES (ref) had very good abrasion resistance, as expected [23], although the mass loss increased, with increased rubbing cycles (up to $0.41 \%$ after 20,000 rubbings). By the repeated rubbing on the textile's surface, the fibres are bent and shifted in the rubbing direction. As the number of rubbing cycles rises, deflection of the fibres increases, as well as the tension, until the point when the filaments finally break [24]. Modification of the fabric by coating with a polymer could change the slip resistance or the friction resistance, and thus, change the fabric's abrasion resistance, depending on the type of coating compound. In this study, the highest mass loss was observed with the AR-coated sample, from 0.73 (10,000 cycles) up to $3.35 \%$ (20,000 cycles). The lowest mass loss was recorded at the PUR-coated sample $(0.04 \%$ up to $0.21 \%)$, indicating a good protection of the fabric surface against rubbing. As explained in [25], PUR offers an outstanding abrasive wear resistance when compared with rubbers, plastics, or even metals, and is therefore widely used in the systems where a high abrasion resistance is required. In the case of the PDMS screen-printed sample a mass loss of up to $0.22 \%$ was observed. Although it was noted that some of the PDMS coating had peeled off during the rubbing test, it remained glued together with the textile surface in the form of beads and therefore the mass was not significantly changed. According to the literature [24], 
seat covers for furniture in private use should withstand 15,000 rubbing cycles without any damage, while those used in workplaces should last for at least 35,000 cycles. Thus, all the polymers from our study could be used as protective coatings, on furniture at least.

\subsubsection{UV Photo-Stability}

Although polymers are believed to be "everlasting materials", they experience some type of degradation during their service life, resulting in an appreciable modification in their properties. Thus, uncoated and PDMS-, PUR-, AR- and ER-coated PES samples were irradiated in an UV chamber with a combination of artificial UVA and UVB lights up to 30 days, in order to study the photo-stability of coatings when applied on the textile material under prolonged UV light exposure. The optical changes of the samples were evaluated before and after irradiance by reflectance measurement, from which CIE whiteness $(W)$ and yellowness (YI) indices were calculated (Equations (2) and (3)) and presented in Figure 5.

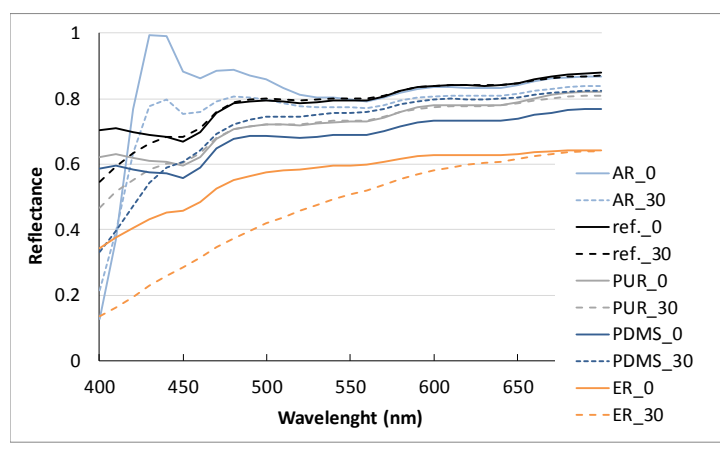

(a)

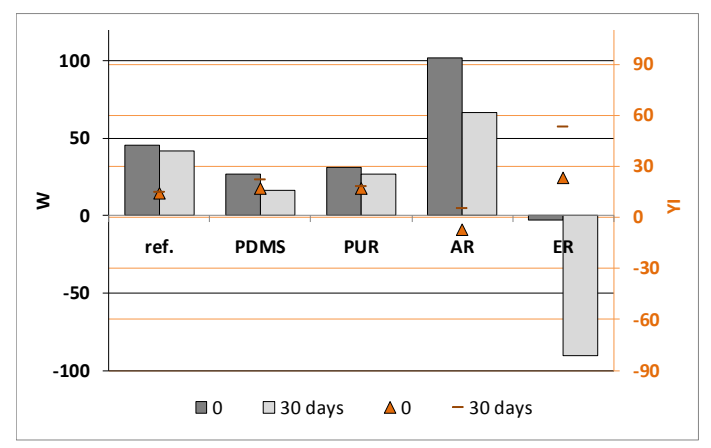

(b)

Figure 5. Samples before 0 and after 30 days of UV light exposure: reflectance curves (a) and $W$ and $Y I$ values $(\mathbf{b})$.

From reflectance curves (Figure 5a) and $W$ and $Y I$ values (Figure $5 b$ ), it could be observed that after ER and AR application, the optical properties of PES drastically changed. ER is yellowish in colour and, despite its transparency and thin application layer, this caused changes in its surface reflectance, as expected.

After several days of UV exposure, ER coated sample became brownish (data not shown), escalating the $Y I$ from 23.15 up to 52.88 in 30 days. This was in contrast to the AR coated sample, which became whiter compared to the reference, due to the content of the fluorescent pigment, which absorbs light in the UV region (wavelengths below $400 \mathrm{~nm}$ ) and reflects it from the surface in the Vis region above $400 \mathrm{~nm}$ (Figure 5a). After 30 days of UV exposure, the whiteness of the AR sample showed a very significant decrease from 102.1 down to 66.5. The application of PDMS and PUR on the PES fabric also changed the reflectance curves (Figure 5a), where the samples became darker in comparison to the reference one. However, after UV irradiation, the reflectance and $W$ and $Y I$ values remained almost the same, which is in agreement with the results obtained by other researchers [26]. Some small changes could be observed between 400 and $440 \mathrm{~nm}$, but this was also seen for the reference sample. From the obtained results, it could be concluded that both PDMS and PUR are photo-stable polymers, which are not prone to ageing, and as such, they would be appropriate for the protection of conductive tracks that would be exposed to the sun or artificial lights.

\subsection{Characterisation of Cu Metallised/Polymer Coated PES}

To evaluate the suitability of the selected polymers to protect electroless plated $\mathrm{Cu}$ on PES textiles against corrosion, $\mathrm{Cu}$ metallised/polymer coated samples were washed for up to 30 cycles and analysed using several complementary approaches. These were: (i) determination of electrical resistivity before 
and after 5, 10, 20, and 30 washing cycles (Table 3), (ii) investigation of optical properties by measuring the fabrics' reflectance before and after 5, 15, and 30 washing cycles, followed by the calculation of CIE total colour differences (Table 4), and (iii) evaluation of surface morphologies before and after 30 cycles by means of OM and SEM (Figure 6). Metallised/polymer coated textiles will be further used to produce assistive technology for older adults (ongoing research).

Table 4. CIE values of samples and differences between $\mathrm{Cu}$ metallised/protected non-washed and washed samples (up to 30 cycles).

\begin{tabular}{|c|c|c|c|c|c|c|c|c|c|c|c|c|c|c|c|c|c|c|}
\hline \multicolumn{7}{|c|}{ CIE $L^{*} a^{*} b^{*}$ Values } & \multirow{2}{*}{\multicolumn{12}{|c|}{$\begin{array}{c}\text { CIE Colour Differences } \\
\text { Washing Cycles }\end{array}$}} \\
\hline \multirow{2}{*}{\multicolumn{3}{|c|}{ Cu Metallized }} & \multirow{2}{*}{\multicolumn{4}{|c|}{ Cu Metallized/Polymer Coated }} & & & & & & & & & & & & \\
\hline & & & & & & & \multicolumn{4}{|c|}{5} & \multicolumn{4}{|c|}{15} & \multicolumn{4}{|c|}{30} \\
\hline $\mathbf{L}^{*}$ & $a^{*}$ & $\mathbf{b}^{*}$ & Polymer & $\mathbf{L}^{*}$ & $a^{*}$ & $\mathbf{b}^{*}$ & $d L^{*}$ & $d a^{*}$ & $d b^{*}$ & $d E^{*}$ & $d L^{*}$ & $d a^{*}$ & $d b^{*}$ & $d E^{*}$ & $d L^{*}$ & $d a^{*}$ & $d b^{*}$ & $d E^{*}$ \\
\hline 42.98 & 14.88 & 13.01 & - & & & & -8.50 & -7.44 & -7.35 & 13.47 & -8.26 & -6.96 & -6.02 & 12.37 & & & & \\
\hline 43.29 & 14.82 & 12.91 & PDMS & 32.67 & 8.11 & 7.48 & 0.53 & -1.58 & -0.77 & 1.84 & -0.47 & -2.90 & -1.16 & 3.16 & 0.13 & -4.00 & -1.06 & 4.13 \\
\hline 43.22 & 15.17 & 13.13 & PUR & 33.70 & 8.35 & 7.96 & 1.97 & -0.42 & -1.33 & 2.41 & 2.06 & -0.62 & -0.48 & 2.21 & 3.63 & -2.01 & -1.50 & 3.30 \\
\hline 44.66 & 14.21 & 15.49 & $\mathrm{AR}$ & 36.25 & 9.82 & -0.19 & 0.97 & -0.56 & 0.57 & 1.25 & -0.05 & -0.88 & 1.68 & 1.90 & 0.52 & -1.68 & -2.79 & 4.41 \\
\hline 44.02 & 14.47 & 12.66 & ER & 35.52 & 7.91 & 4.24 & 0.44 & 0.04 & 1.97 & 2.02 & 1.17 & -1.12 & 2.49 & 2.97 & & & & \\
\hline
\end{tabular}

$\mathrm{Cu} / \mathrm{PDMS}$
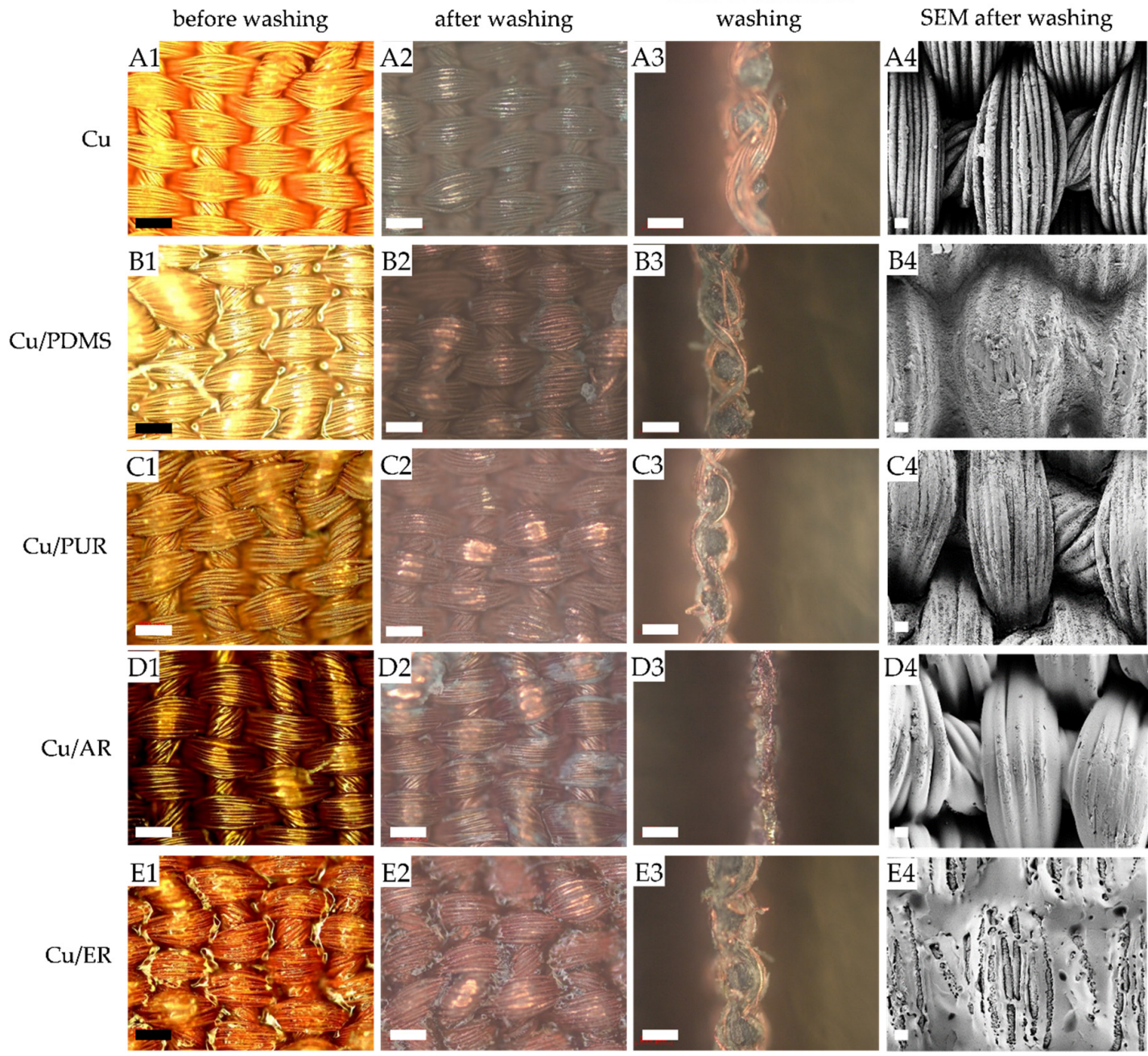

Cross-section after
washing

Figure 6. OM of $\mathrm{Cu}$ metallised/protected samples: before washing (A1-E1); after washings (A2-E2); cross-section after washings (A3-E3); and SEM after washings (A4-E4); Cu (A1-A4); Cu/PDMS (B1-B4); Cu/PUR (C1-C4); Cu/AR (D1-D4); Cu/ER (E1-E4). Scale bar: $200 \mu \mathrm{m}$ for OM and $20 \mu \mathrm{m}$ for SEM. 


\subsubsection{Electrical Resistivity}

Electrical resistance of material indicates how strongly the material opposes the flow of electric current through it. In the case of conductive textiles, the resistance is affected by the uniformity of electroless copper deposit, the arrangement of fibres and yarns in the textiles, the geometrical dimensions of the sample and on its structure [27]. The electrical resistivity of samples was measured between two points at three measuring lines/distances $(1,2,3)$ on each sample, as presented on Figure 2, which allowed the assessment of the conductive properties of the whole textile sample surface. Each sample was measured after electroless copper deposition and then again after protection ( $\mathrm{Cu}$ metallised/polymer coated) and after the 5th, 10th, 20th and 30th washings (Table 3).

It should be noted from Table 4 that the electrical resistances of the $\mathrm{Cu}$ metallised samples were in the range from 0.1 up to $1.5 \Omega$, suggesting good conductivity of samples. It is also apparent that the electrical resistivity varied for the same sample, depending on the measuring line/distance, since, compared with other engineering materials, textiles are not homogeneous and anisotropic products. Specific surface and physical-mechanical properties of the textile material, such as elasticity, bending and stretching, influence the uniform coating of the electroless $\mathrm{Cu}$ deposit across the whole surface.

As explained by Tokarska et al. [27], the sample resistance is associated with contact resistance between neighbouring yarns and numerous contact points where yarns in the sample cross (different number of warp and weft yarns per length unit). Depending on where the electrodes are located, current flows in a different way (the path of easiest flow) in the fibrous structure. Table 3 shows that after application of the polymers, electrical resistivity increased (conductivity decreased), depending on the initial electrical resistivity, measuring line/distance, and employed polymer. The reference sample, which was not protected with polymer, recorded the highest electrical resistivity after 10 washing cycles (up to $1 \mathrm{M} \Omega$ ), and thus, it was no longer conductive. The same trend was observed at the ER protected sample after 10 washing cycles, where the electrical resistivity was measured at $65.3 \mathrm{k} \Omega$, revealing the least effective protection against water as compared with other polymer' protected samples. During washing, the ER film formation on the surface cracked, as could be further seen from the SEM micrograph (Figure 6), and the water easily penetrates between the pores, causing a loss of conductivity. The three remaining samples were still conductive after 30 washing cycles, in the following order from the highest to the lowest: AR, PDMS and PUR, providing successful protection of the Cu metallised surfaces against water, detergent and mechanical forces during washing.

\subsubsection{Colour Evaluation of Samples}

Samples were calorimetrically evaluated by measuring the reflectance, from which the CIE L*a* $b^{*}$ values were calculated. This allows for an analysis of how the optical properties are affected by the coatings, as well as the effect of laundering cycles on possible colour changes (eventual corrosion). Furthermore, the colour differences in lightness $\left(d L^{*}\right)$, on $\mathrm{a}^{*}-\mathrm{b}^{*}$ axis $\left(d a^{*}\right.$ and $\left.d b^{*}\right)$ and total colour differences $\left(d E^{*}\right)$ were determined between $\mathrm{Cu}$ metallised and $\mathrm{Cu}$-metallised/protected textiles plus between the non-washed and washed samples, after 5, 15 and 30 washing cycles (Table 4).

It is evident, from Table 4, that each of the five electroless $\mathrm{Cu}$ plated samples had a slightly different colour ( $\mathrm{a}^{*}$ and $\mathrm{b}^{*}$ axes), as well as lightness ( $\mathrm{L}^{*}$ axis). After the application of the protective coatings, the lightness drastically decreased, irrespectively of the polymer used, implying darker hues, as reported in a previous study [17]. Moreover, all samples became less red and less yellow (greener and bluer) in comparison to the unprotected fabrics, as could also be perceived from the OM images (Figure 6) and determined from the reflectance values in Figure 5a. After 5 washing cycles, the most significant changes in hue (where the sample became more greenish-blue) and lightness occurred on the Cu-metallised sample that was not protected with polymer; i.e., $d E^{*}$ was 13.47 , revealing that corrosion had already started. On the other hand, the $d E^{*}$ of PDMS, ER, PUR and AR protected samples increased to 2.41 after 5 cycles, up to 3.41 after 15 cycles and up to 4.41 after 30 cycles, depending on the employed polymer. These changes are not linear, and they are dependent on the uniformity of $\mathrm{Cu}$ deposition and polymer coatings, as well as the location of the measuring area on an individual sample. 


\subsubsection{Surface Observation by OM and SEM}

The surfaces, cross-sections, and surface morphologies of $\mathrm{Cu}$-metallised and $\mathrm{Cu}$-metallised/polymer protected samples, before and after washings, were inspected in detail by OM and SEM techniques (Figure 6).

Figure 6A1-A4 displays a uniform and dense Cu deposit on the surface of PES fabric obtained by electroless plating, wrapping the entire surface of an individual fibre. The polymers changed the surfaces of the fabrics in that they became smoother, although the $\mathrm{Cu}$ deposit is still visible, because of the transparency of the colourless polymers. It is also evident from OM and SEM images that PDMS and ER filled the pores between fibres, forming thicker films on the surfaces as compared to PUR and AR coatings, which agrees with the results in Table 2. In addition, all polymers remained on the surfaces of samples after 30 washing cycles (as shown in Figure 6A2-E2,A4-E4)), although some deterioration/cracking of the polymer film can be observed, especially at ER, due to the combined function of water, auxiliaries and mechanical forces. In general, all polymers on the coated samples become visually less bright after intensive washings as they are slightly shaded, but still yellow-reddish in comparison to the corroded green-blueish un-coated reference, as also confirmed by colour measurement (Table 4). AR presents the most homogeneous coating after 30 washing cycles (Figure 6D1-D4), exhibiting the best protection of the $\mathrm{Cu}$ deposit, which agrees with the electrical resistivity results presented in Table 3 .

\section{Conclusions}

In the first part of this study, four commercially available polymers were applied on PES fabric according to pre-optimised screen-printing or padding processes and comprehensively characterised, in order to evaluate their effect on the physical-mechanical properties of the base textile. Moreover, the wear and photo resistances of polymer coatings were evaluated. The results obtained by the physical-mechanical characterisation of coated PES demonstrated that PUR and AR had less influence on air permeability, tensile strength and breaking tenacity when compared to PDMS and ER. On the other hand, PUR and PDMS had the highest abrasion resistance, with mass loss of $0.21 \%$ and $0.22 \%$ at 20,000 rubbing cycles, and superior photo-stability under prolonged UV irradiation in comparison with AR and ER.

In the second part of the study, a PES fabric was successfully $\mathrm{Cu}$ coated using a Pd-catalysed $\mathrm{Cu}$ electroless plating process, as confirmed by SEM images and electrical resistivity measurement. This electroless $\mathrm{Cu}$ deposit was then protected using the four selected polymers. The results demonstrated that AR, PDMS and PUR imparted durable protection to $\mathrm{Cu}$ against 30 washing cycles, while simultaneously retaining good conductivity of the fabrics. It was found that the colour of the deposit did not change noticeably, thereby implying good corrosion protection. When considering the results of all examined parameters, PUR applied by pad-drying technique presented the most suitable protection of $\mathrm{Cu}$ tracks on PES, with the lowest impact on physical-mechanical properties of base textiles. On the other hand, ER, which is commonly employed in the printed circuit boards industry, is not recommended to be used for protection of $\mathrm{Cu}$ tracks on fabrics, due to its rigidity, low photo-stability and washing durability. The obtained results are a part of an ongoing project, and will be further used in the production of assistive technology for older-adults in 3 specific areas, namely (i) footwear: insoles that can analyse gait and warn of falls, (ii) furniture: sofa with the ability to monitor breathing and activity and (iii) clothing: $t$-shirt with integrated heaters that can detect body temperature and warm the user if they are cold.

Author Contributions: Conceptualization, A.O. and A.C.; methodology, A.O., D.F. and L.K.; formal analysis, N.V., D.F. and L.K.; investigation, A.O., N.V., D.F., L.K. and A.C.; data curation, A.O., N.V., D.F. and L.K.; writing-original draft preparation, A.O. and D.F.; writing-review and editing, A.O., D.F., L.K. and A.C.; supervision, A.O. and A.C.; funding acquisition, A.O. and A.C. All authors have read and agreed to the published version of the manuscript. 
Funding: The MATUROLIFE project leading to this research has received funding from the European Union's Horizon 2020 research and innovation program, under grant agreement No 760789.

Conflicts of Interest: The authors declare no conflict of interest.

\section{References}

1. Govaert, F.; Vanneste, M. Preparation and Application of Conductive Textile Coatings Filled with Honeycomb Structured Carbon Nanotubes. J. Nanomater. 2014, 2014, 6-12. [CrossRef]

2. Ghosh, S. Electroless copper deposition: A critical review. Thin Solid Film. 2019, 669, 641-658. [CrossRef]

3. Wills, K.A.; Krzyzak, K.; Bush, J.; Ashayer-Soltani, R.; Graves, J.E.; Hunt, C.; Cobley, A.J. Additive process for patterned metallized conductive tracks on cotton with applications in smart textiles. J. Text. Inst. 2018, 109, 268-277. [CrossRef]

4. Graves, J.E.; Sugden, M.; Litchfield, R.E.; Hutt, D.A.; Mason, T.J.; Cobley, A.J. Ultrasound assisted dispersal of a copper nanopowder for electroless copper activation. Ultrason. Sonochemistry 2016, 29, 428-438. [CrossRef]

5. Litchfield, R.E.; Graves, J.E.; Sugden, M.A.; Hutt, D.E.; Cobley, A. Functionalised copper nanoparticles as catalysts for electroless plating. In Proceedings of the 2014 IEEE 16th Electronic Packaging Technology Conference, Singapore, 3-5 December 2014; pp. 235-240.

6. Paniwynk, L.; Cobley, A. Ultrasonic Surface Modification of Electronics Materials. Phys. Procedia 2010, 3, 1103-1108. [CrossRef]

7. Al-Maqdasi, Z.; Hajlane, A.; Renbi, A.; Ouarga, A.; Chouhan, S.S.; Joffe, R. Conductive Regenerated Cellulose Fibers by Electroless Plating. Fibres 2019, 7, 38. [CrossRef]

8. Cui, X.; Hutt, D.A.; Scurr, D.J.; Conway, P.P. The evolution of Pd/Sn catalytic surfaces in electroless copper deposition. J. Electrochem. Soc. 2011, 158, D172-D177. [CrossRef]

9. Liu, X.; Guo, R.; Shi, Y.; Deng, L.; Li, Y. Durable, Washable, and Flexible Conductive PET Fabrics Designed by Fiber Interfacial Molecular Engineering. Macromol. Mater. Eng. 2016, 301, 1383-1389. [CrossRef]

10. Shahariar, H.; Kim, I.; Soewardiman, H.; Jur, J.S. Inkjet Printing of Reactive Silver Ink on Textiles. ACS Appl. Mater. Interfaces 2019, 11, 6208-6216. [CrossRef]

11. Zhu, C.; Li, Y.; Liu, X. Polymer interface molecular engineering for E-textiles. Polymers 2018, 10, 573. [CrossRef]

12. Cho, J.; Moon, J.; Jeong, K.; Cho, G. Application of PU-sealing into Cu/Ni electroless plated polyester fabrics for e-textiles. Fibers Polym. 2007, 8, 330-334. [CrossRef]

13. Baldissera, A.F.; Ferreira, C.A. Coatings based on electronic conducting polymers for corrosion protection of metals. Prog. Org. Coat. 2012, 75, 241-247. [CrossRef]

14. Kazani, I.; Hertleer, C.; De Mey, G.; Schwarz, A.; Guxho, G.; Van Langenhove, L. Electrical Conductive Textiles Obtained by Screen Printing. Fibres Text. East. Eur. 2012, 20, 57-63.

15. Yang, K.; Torah, R.; Wei, Y.; Beeby, S.; Tudor, J. Waterproof and durable screen printed silver conductive tracks on textiles. Text. Res. J. 2013, 83, 2023-2031. [CrossRef]

16. Paul, G.; Torah, R.; Beeby, S.; Tudor, J. The development of screen printed conductive networks on textiles for biopotential monitoring applications. Sens. Actuators A Phys. 2014, 206, 35-41. [CrossRef]

17. Jiang, S.; Miao, D.; Li, A.; Guo, R.; Shang, S. Adhesion and Durability of Cu Film on Polyester Fabric Prepared by Finishing Treatment with Polyester-polyurethane and Aqueous Acrylate. Fibers Polym. 2016, 17, 1397-1402. [CrossRef]

18. Fakin, D.; Stana-Kleinschek, K.; Kurečič, M.; Ojstršek, A. Effects of nanoTiO $2-\mathrm{SiO}_{2}$ on the hidrophilicity/dyeability of polyester fabric and photostability of disperse dyes under UV irradiation. Surf. Coat. Technol. 2014, 253, 185-193. [CrossRef]

19. Kara, S.; Yeşilpinar, S.; Akşit, A. Permeability properties and abrasion resistance of coated polypropylene fabrics. Fibres Text. 2018, 2, 40-47.

20. Moiz, A.; Vijayan, A.; Padhye, R.; Wang, X. Chemical and water protective surface on cotton fabric by pad-knife-pad coating of WPU-PDMS-TMS. Cellulose 2016, 23, 3377-3388. [CrossRef]

21. Lee, S.; Park, C.H. Conductivity, superhydrophobicity and mechanical properties of cotton fabric treated with polypyrrole by in-situ polymerization using the binary oxidants ammonium Peroxodisulfate and ferric chloride. Text. Res. J. 2019, 89, 2376-2394. [CrossRef] 
22. Bulut, Y.; Suülar, V. Effects of process parameters on mechanical properties of coated fabrics. Int. J. Cloth. Sci. Technol. 2011, 23, 205-221. [CrossRef]

23. Somogyi Škoc, M.; Pezelj, E. Abrasion Resistance of High. Performance Fabrics. In Abrasion Resistance of Materials; Marcin, A., Ed.; IntechOpen: London, UK, 2012.

24. Textor, T.; Derksen, L.; Bahners, T.; Gutmann, J.S.; Mayer-Gall, T. Abrasion resistance of textiles: Gaining insight into the damaging mechanisms of different test procedures. J. Eng. Fibers Fabr. 2019, 14, 1558925019829481. [CrossRef]

25. Kwiatkowski, K.; Nachman, M. The Abrasive Wear Resistance of the Segmented Linear Polyurethane Elastomers Based on a Variety of Polyols as Soft Segments. Polymers 2017, 9, 705. [CrossRef] [PubMed]

26. Israeli, Y.; Lacoste, J.; Cavezzad, J.; Lemaire, J. Photooxidation of polydimethylsiloxane oils and resins. IV-effect of phenyl groups. Polym. Degrad. Stab. 1995, 47, 357-362. [CrossRef]

27. Tokarska, M.; Frydrysiak, M.; Zięba, J. Electrical properties of flat textile material as inhomegeneous and anisotropic structure. J. Mater. Sci. Mater. Electron. 2013, 24, 5061-5068. [CrossRef]

(C) 2020 by the authors. Licensee MDPI, Basel, Switzerland. This article is an open access article distributed under the terms and conditions of the Creative Commons Attribution (CC BY) license (http://creativecommons.org/licenses/by/4.0/). 